\title{
Zielklärung und Zielerreichung im Coaching
}

\section{Ergebnisse einer qualitativen Untersuchung von Coaching-Prozessen}

\author{
Monika Wastian ${ }^{1} \cdot$ Janina Poetschki $^{1}$
}

Online publiziert: 24. Mai 2016

(C) The Author(s) 2016. This article is available at SpringerLink with Open Access

Zusammenfassung In einer qualitativen Untersuchung wurden Coaching-Prozesse mit Hilfe einer visuell gestützten Interview-Methode mit 43 Coachs bzw. 19 Klienten rekonstruiert, um unter anderem den Prozess der Zielklärung und Zielerreichung zu explorieren. Dabei gingen wir den folgenden Fragen nach: a) Wie erfolgen die Zielklärung sowie die Steuerung der Zielfortschritte im Coaching aus der Sicht von Coaches bzw. Coaching-Klienten und inwieweit werden dabei die Wirkzusammenhänge der Zielsetzungstheorie berücksichtigt? b) Wann im Verlauf des Coachings werden die Ziele zwischen den Beteiligten thematisiert? c) Gibt es typische Muster des Zielklärungsbzw. Zielerreichungsprozesses?

Wie die Ergebnisse zeigen, werden Coaching-Ziele während des gesamten Coaching-Prozesses und teilweise sogar darüber hinaus im Rahmen von Follow-ups thematisiert, doch werden sie mehrheitlich nicht spezifisch, messbar und terminiert geklärt. Die Sichtweisen von Coachs und Coachees klaffen diesbezüglich deutlich auseinander. Dabei nennen Coachees spezifische und messbare Ziele als wichtigsten Verbesserungswunsch. Als typischste Muster ließen sich die SMARTe Zielklärung zu Beginn des Coachings und

Dieser Beitrag entspricht, in leicht abgewandelter Form dem Kapitel: Wastian, M. \& Poetschki, J. (2016). Coaching-Ziele klären und erreichen: Wie Coachs ihre Coachees zum Erfolg führen. In C. Triebel, J. Heller, B. Hauser \& A. Koch (Hrsg.), Qualitätsmanagement im Coaching. Berlin, Heidelberg: Springer.

- Wir danken den Herausgebern und dem Verlag für die Druckgenehmigung.

Monika Wastian

info@inforp.com

1 Institut für Organisationspsychologie, 80453 München, Deutschland die Zielklärung als „Suchlauf“ über den Coaching-Prozess hinweg identifizieren.

Implikationen für die Coaching-Forschung und Praxis werden diskutiert und Handlungsempfehlungen gegeben, wie Coaching-Beteiligte zur Qualität und zum Erfolg ihrer Coachings beitragen können.

Schlüsselwörter Zielklärung · Evaluation · Zielerreichung · Coaching-Wirkfaktoren · Qualitätssicherung im Coaching · Feedback

\section{Goal setting and goal attainment in coachings}

Results of a qualitative study on coaching processes

Abstract This qualitative study with 43 coaches and 19 coaching clients used a graphically supported interviewing method to reconstruct coaching processes and to explore goal setting and goal achievement during coaching processes. Research questions were as follows: a) How are coaching goals clarified and how are goal progress and achievement controlled from coaches' or, respectively, coachees' point of views? To which extent do coaches consider success factors of goal setting theory? b) At what time do coaches and coachees raise the issue of coaching goals? c) Are there typical patterns of goal setting or goal achievement processes?

Results show that coaching goals are brought up throughout the coaching process or beyond, during follow ups. However, most of the goals are not set in a specific, measurable and scheduled way - even more so from the coachees' point of view. Specific and measurable goals are the major improvement demanded by coachees. Typical goal setting patterns were the clarification of SMART goals at the be- 
ginning of the coaching or a continuous process of goal clarification and adaption throughout the coaching.

We discuss implications for coaching research and practice and recommend actions coaching participants can take to improve the quality and success of their coachings.

Keywords Goal setting - Evaluation - Goal achievement · Coaching success factors $\cdot$ Coaching quality $\cdot$ Feedback

\section{Einleitung}

Bis vor wenigen Jahren gab es zwar eine wachsende Anzahl von Untersuchungen, welche die Wirksamkeit von Coachings bestätigte. Es ließ sich jedoch kaum empirisch begründen, wodurch Coaching wirkte und wie sich die Qualität von Coachings beeinflussen lässt, denn es fehlte noch an Studien zu Coaching-Prozessen (vgl. Greif 2008). Aus diesem Grunde initiierten wir im Jahr 2007 das Forschungsprojekt „Qualitätssicherung im Coaching“, das bis 2012 bei uns am Institut für Organisationspsychologie in München in Kooperation mit der Ludwig-Maximilians-Universität durchgeführt wurde. Das Wissen, was Coaching-Prozesse erfolgreich macht und wie sich die Coaching-Qualität beeinflussen lässt, sollte Coaching-Beteiligten, Personalentwicklern und Weiterbildungseinrichtungen die Grundlage für ein Qualitätsmanagement im Coaching bzw. für die Aus- und Weiterbildung von Coachs liefern. Unter anderem sollten durch das Forschungsprojekt Erfolg versprechende Prozesse, Methoden, Strategien und Kriterien im Hinblick auf

- die Coach-Auswahl,

- Aufbau und Verwaltung eines Coach-Pools,

- Coaching-Interventionen,

- Rahmenbedingungen für Coaching-Prozesse,

- Coaching-Anwendung, -Systematisierung, -Qualitätssicherung.

identifiziert werden. Hierzu gehörten auch Maßnahmen zur Zielklärung sowie zur Steuerung und Evaluation von Zielfortschritten.

\section{Zielsetzungstheorie und SMARTe Coaching- Ziele}

Mittlerweile liegen einschlägige Nachweise zur Effektivität von Coaching vor (Grant et al. 2010; Theeboom et al. 2013) und zunehmend werden dabei auch Wirkfaktoren im Coaching diskutiert und untersucht (Jones et al. 2015; Künzli 2009). Soweit sie spezifische Coaching-Interventionen betreffen, ist die Befundlage zwar noch dünn, doch es kann davon ausgegangen werden, dass zielbezogene Interventio- nen wichtige Wirkfaktoren darstellen (siehe z. B. Greif et al. 2012).

Nicht nur für Mitarbeitergespräche (Winkler und Hofbauer 2010), für die Führung (Schmidt und Kleinbeck 2006) und Projektarbeit (Wastian et al. 2015), sondern auch für Coachings (Greif 2008) wird empfohlen, Ziele SMART zu klären, d.h. spezifisch, messbar, attraktiv oder akzeptiert, realistisch und terminiert.

Hinter der SMART-Formel verbirgt sich die vielfach empirisch bestätigte Zielsetzungstheorie von Locke und Latham (Locke und Latham 1990, 2002). Demnach fördern spezifische und erreichbare, aber herausfordernde Ziele (entsprechend dem S, M, T bzw. dem R in der SMARTFormel) die individuelle Leistung und Zielerreichung, wenn eine Person Feedback zu ihren Zielfortschritten bekommt z. B. durch den Coach, durch eigene Fortschrittskontrollen oder durch Personen in ihrem Arbeits- und Lebensumfeld - und wenn sie sich an das Ziel gebunden fühlt. Sie muss das Ziel also akzeptieren und attraktiv finden (entsprechend dem A in der SMART-Formel). Auf diese Weise ist es möglich, einen Hochleistungszyklus (vgl. Locke und Latham 2002) in Gang zu setzen: Die eigene Leistung und Zielerreichung tragen zur Zufriedenheit und zu lohnenden Ergebnissen für die Person bei, was sie dazu anspornt, weitere Herausforderungen anzunehmen und sich an noch größere Ziele zu binden (A in der SMART-Formel). Abb. 1 stellt diese Zusammenhänge grafisch dar.

Diese Zusammenhänge bestätigen zahlreichen Untersuchungen (Überblick: Locke und Latham 1990, 2002; Seijts et al. 2004). Eine so vorgenommene Zielsetzung wird deshalb seit langem als erfolgreiche Motivationstechnik in den unterschiedlichsten Bereichen eingesetzt, u. a. in Trainings.

Klein et al. (1999) befassten sich in ihrer Meta-Analyse mit der Wichtigkeit der Zielbindung in einem Zielklärungsprozess. Die Untersuchungen ergaben, dass ein positiver Zusammenhang zwischen Zielbindung und Leistung besteht. Das bedeutet, dass wenn ein Individuum sich beispielsweise ein Leistungsziel setzt, welches von ihm selbst akzeptiert und angenommen wird, die Leistung höher ausfällt, als bei Fehlen von Zielbindung. Eine weitere MetaAnalyse ergab (Neubert 1998), dass der Effekt umso größer wird, je schwieriger die zu bewältigende Aufgabe bzw. das Ziel ist. Des Weiteren beweisen die Ergebnisse, dass die Leistung durch spezifische und herausfordernde Zielsetzungen und Feedback erhöht wird. Es konnte auch gezeigt werden, dass bei Hinzufügen von Feedback eine deutlich höhere Wirkung eintritt, wenn die Aufgaben komplex und herausfordernd sind (ebenda). Entsprechend kann das Feedback durch den Coach die Effektivität von Coachings unterstützen (Passmore und Fillery-Travis 2011).

Johnson et al. (2012) untersuchten die Wirkung von Zielsetzungen im Hinblick auf eine mögliche verbesserte Transferleistung nach Trainings. Die Forschungsergebnisse ver- 
Abb. 1 Hochleistungszyklus

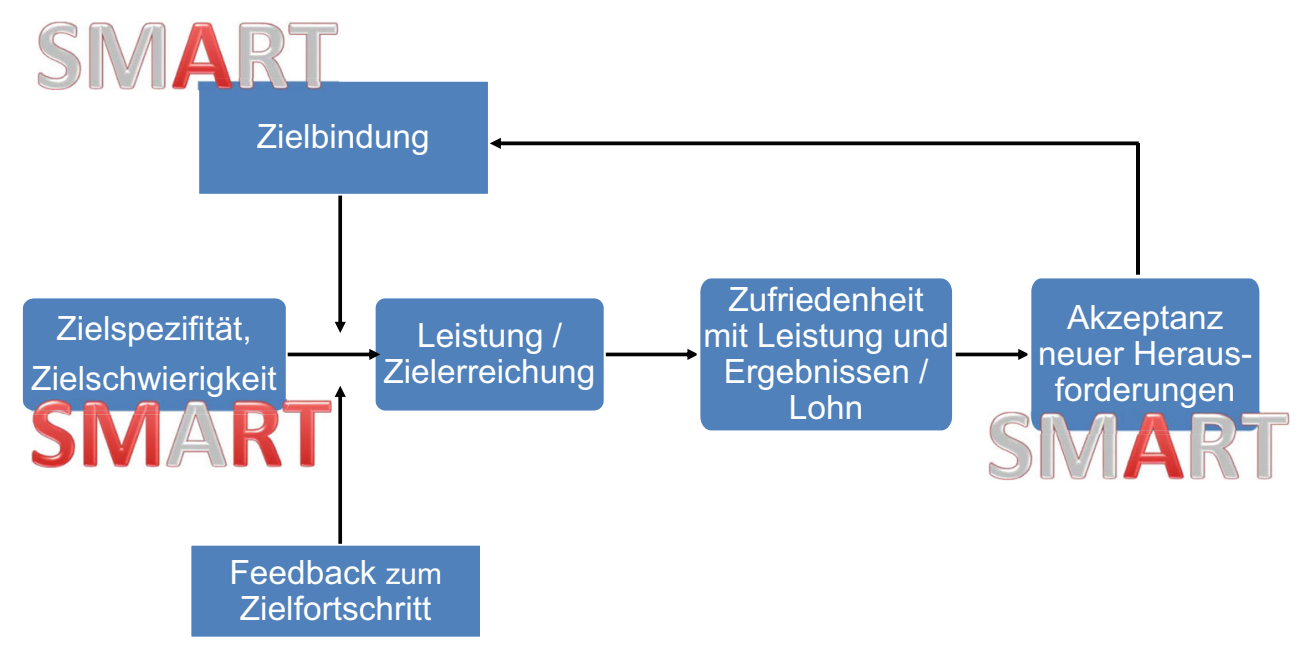

deutlichen, dass Zielsetzungen die Zielbindung, die Motivation und die Beharrlichkeit bei der Zielverfolgung erhöhen. Darüber hinaus nahmen die trainierten Führungskräfte eine Veränderung ihres Führungsverhaltens entsprechend den Zielen wahr, die sie sich gesetzt hatten (ebenda).

Insgesamt sprechen Erkenntnisse zur Zielsetzungstheorie also dafür, dass eine SMARTe Zielklärung eine wirksame Coaching-Intervention sein könnte. Dies sagen auch Befunde aus der Psychotherapieforschung (u. a. Grawe et al. 1994), aus denen Greif und Kollegen $(2008 ; 2012)$ ein Wirkfaktoren-Modell für das Coaching abgeleitet haben. $\mathrm{Zu}$ den Wirkfaktoren, die zum Coaching-Erfolg beitragen, gehören ihren Erkenntnissen zufolge die Zielklärung (beispielsweise anhand der SMART-Kriterien), die Ressourcenaktivierung und die Umsetzungsunterstützung. Letzt genannte beinhalten die Identifizierung klienteneigener Ressourcen und die Entwicklung von Umsetzungsplänen zur Zielerreichung. Die Evaluation der Zielfortschritte während des Coachings ermöglicht eventuelle Änderungen der Zielverfolgung während des Coachings und gestattet zu jeder Zeit eine Kontrolle des Zielerreichungsgrades von Seiten des Coachs sowie des Coachees.

Mit Blick auf den Forschungsstand zur Zielsetzungstheorie und den potenziellen Wirkfaktoren im Coaching lässt sich schlussfolgern, dass es nützlich ist, Ziele SMART zu klären und Zielfortschritte zu beobachten, rück zu melden und zu steuern. Aber wie verfahren Coachs und Coachees dabei tatsächlich und welche Vorgehensweise ist wirklich „SMART“? Um dies herauszufinden, haben wir uns in der Studie „Qualitätssicherung im Coaching“ u. a. damit beschäftigt wie Ziele in erfolgreichen Coaching-Prozessen geklärt und erreicht werden.

\section{Forschungsfragen}

Ausgehend von der Annahme, dass die Zielklärung einen wichtigen Wirkfaktor im Coaching darstellt (Greif 2008) und dass SMARTe Ziele zur Leistung und Zielerreichung beitragen (entsprechend der Zielsetzungstheorie von Locke und Latham 1990, 2002), wurde untersucht, wie Coachs im Verlauf erfolgreicher Coaching-Prozesse die Ziele ihrer Klienten klären bzw. wie sie Zielfortschritte und die Zielerreichung steuern, kontrollieren und evaluieren. Dabei wurde den folgenden Fragen nachgegangen:

a) Wie wird der Weg zum Ziel geklärt, gesteuert und kontrolliert und inwieweit findet die SMART-Formel dabei Anwendung? Inwieweit unterscheiden sich diesbezüglich die Aussagen von Coachees und Coachs?

b) Wann werden im Coaching-Prozess Ziele zwischen Coach und Coachee thematisiert?

c) Lassen sich typische Muster des Zielklärungs- bzw. -erreichungsprozesses erkennen?

\section{Untersuchungsdesign und -methode}

\subsection{Design und Sample}

Ziel des Projektes „Qualitätssicherung im Coaching“, in deren Rahmen den Forschungsfragen nachgegangen wurde, war es, Coaching-Beteiligten - Coachs, Coachees und ggf. deren Führungskräften sowie Personalentwicklern - Wissen zu Erfolgsfaktoren im Coaching und Ansätze zur Qualitätssicherung und zum Qualitätsmanagement im Coaching zu liefern. Dazu war es notwendig, Coaching-Prozesse mit Hilfe qualitativer Methoden vollständig $-\mathrm{d}$. h. von der Coaching-Anfrage über die Durchführung bis zum Follow-up von Coachings - zu untersuchen und die Perspektiven der verschiedenen Beteiligten mit einzubeziehen. 


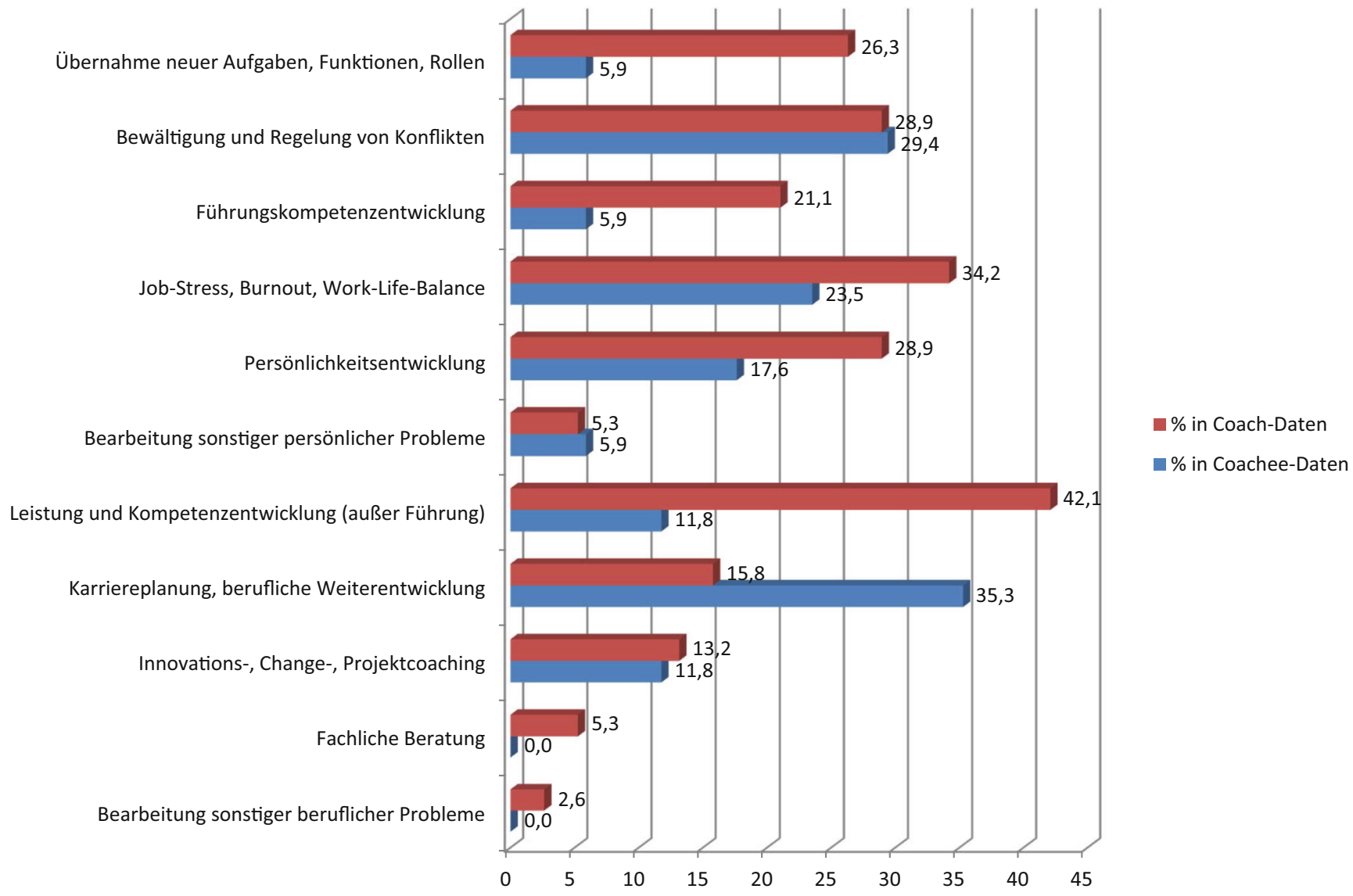

Abb. 2 Anliegen in den geschilderten Coachings

Deshalb interviewten wir u. a. 43 Coachs und 19 Klienten, um abgeschlossene Coaching-Prozesse zu rekonstruieren. Ausgewertet wurden nur Interviews zu vollständig geschilderten Einzelcoaching-Prozessen. Interviews mit fehlenden Daten (z. B. technischer Defekt bei der Aufzeichnung oder Verweigerung der Aufzeichnung) sowie Teamcoachings wurden ausgeschlossen, so dass schließlich 42 Coach-Interviews und 16 Coachee-Interviews zur Auswertung kamen.

Die Coaches wurden aus dem Coach-Netzwerk des Instituts für Organisationspsychologie oder aus Empfehlungen von Coaching-Verantwortlichen in Organisationen rekrutiert. Auf Empfehlung der Coaching-Verantwortlichen sowie der interviewten Coaches erfolgte die Rekrutierung der Coachees.

Die befragten Coaches waren zwischen 33 und 80 Jahre alt $(M=52)$ und hatten zwischen 3 bis 31 Jahren CoachingErfahrung $(M=9,3)$. Bei den Coaches lag der Frauenanteil bei $50 \%$, bei den Coachees waren es 37,5\%. Das Alter der Coachees lag zwischen 24 und 56 Jahren $(M=43)$. 56,3\% waren Führungskräfte, 37,5\% Fachkräfte und 6,3\% gaben sonstige Funktionen an. Abb. 2 zeigt die Verteilung der Klientenanliegen in den geschilderten Coachings, wobei Mehrfachzuordnungen möglich waren.

\subsection{Datenerhebung}

Mit den Interviewpartnern wurden face to face ca. 2stündige Zeitstrahl-Interviews (Wastian und Schneider 2005) durchgeführt. Hierbei handelt es sich um eine auf klientenzentrierter Gesprächsführung (Rogers 2005) und Visualisierung basierte, teilstrukturierte Interview-Methode zur Analyse von Prozessen und erfolgskritischen Ereignissen (Flanagan 1954) im Zeitverlauf. Unterstützt durch eine Zeitstrahlgrafik schildern die Interviewpartner dabei den Verlauf eines erfolgreichen bzw. ihres eigenen Coaching-Prozesses, einschließlich erfolgskritischer Ereignisse, Vorgehensweisen und möglicher Ansatzpunkte für Verbesserungen. Anhand der Zeitstrahl-Grafik werden die erfolgskritischen Ereignisse in mehreren Durchläufen exploriert, wobei sowohl die Grafik als auch klientenzentrierte Gesprächstechniken dazu beitragen, das Erinnerungsvermögen der Interviewpartners anzuregen und ihren Erzählfluss ohne Druck steuern zu können (z. B. durch Paraphrasierung und grafische Fokussierung relevanter Ereignisse). Nach einleitenden teilstrukturierten Fragen (z. B. zum Hintergrund des Coaching-Falls) sind die typischen Schritte beim Zeitstrahl-Interview wie folgt: 
Tab. 1 SMARTe Ziele bzw. Zielfortschritte klären (Poetschki und Wastian 2014)

\begin{tabular}{|c|c|c|c|}
\hline SMART & Zielsetzungstheorie & Definition & $\begin{array}{l}\text { Beispiel-Zitate, die zeigen, wie der Coach die SMART-Kriteri- } \\
\text { en anwendet/thematisiert: }\end{array}$ \\
\hline $\mathrm{S}=$ Spezifisch & Zielspezifität & $\begin{array}{l}\text { Der Coach hinterfragt die spezifi- } \\
\text { schen Kriterien für die Zielerrei- } \\
\text { chung des Coachees }\end{array}$ & $\begin{array}{l}\text { „Ich frage: ,Was wollen Sie erreichen?‘ Und dieses Anliegen } \\
\text { formulieren wir dann durch ständiges Hinterfragen in ein hilf- } \\
\text { reiches Ziel für den Coachee.“ } \\
\text { „Dann sagt der: ,Vom Getriebenen zum Initiator werden ‘[...] } \\
\text { aber wie formuliere ich das als hilfreiches Ziel? ,Keine Zeit } \\
\text { mit Unwesentlichem vertan zu haben“. Wie formuliere ich } \\
\text { das? [...] da ist ganz viel Übersetzungsarbeit notwendigt.“” }\end{array}$ \\
\hline $\mathrm{M}=$ Messbar & Zielspezifität & $\begin{array}{l}\text { Es werden qualitative oder quan- } \\
\text { titative Kriterien vereinbart, an- } \\
\text { hand derer Coach und Coachee } \\
\text { Veränderungen und Fortschritte } \\
\text { im Hinblick auf ein spezifisches } \\
\text { Ziel beobachten/messen können }\end{array}$ & $\begin{array}{l}\text { „Es gibt eh alle drei Monate eine Zielskalierung, eine Über- } \\
\text { prüfung: Wo stehe ich?““ } \\
\text { „Wie viel Zeit wäre denn für ihn ein Zeitgewinn? [...] Woran } \\
\text { würde er das festmachen, dass es für ihn messbar und greif- } \\
\text { bar ist. Sonst kann er hinterher immer sagen, es hat nicht ge- } \\
\text { klappt.“ }\end{array}$ \\
\hline $\begin{array}{l}\text { A = Attraktiv, } \\
\text { Akzeptiert }\end{array}$ & Zielbindung & $\begin{array}{l}\text { Der Coach hinterfragt, ob der } \\
\text { Coachee das Ziel akzeptiert und } \\
\text { positiv bewertet }\end{array}$ & $\begin{array}{l}\text { „Es ist das Ziel des Coachees. Es muss sich für ihn rund an- } \\
\text { fühlen. Er muss es gerne aussprechen.“ } \\
\text { „Wir haben [die Ziele] genauso aufgeschrieben, wie er es ge- } \\
\text { sagt hat [...] nicht mehr und nicht weniger. Denn das war erst- } \\
\text { mal sein Auftrag. Das lasse ich auch so stehen fürs Erste. Es } \\
\text { geht ja nicht darum, dass wir auf so einem Kaugummi lange } \\
\text { rumkauen, bis wir eine dem Coach angenehme Zieldefinition } \\
\text { bekommen.“ }\end{array}$ \\
\hline $\mathrm{R}=$ Realistisch & Zielschwierigkeit & $\begin{array}{l}\text { Der Coach hinterfragt die Wahr- } \\
\text { scheinlichkeit der Zielerreichung, } \\
\text { die Voraussetzungen hierfür so- } \\
\text { wie die Schritte zum Ziel oder er } \\
\text { korrigiert unrealistische Ziele }\end{array}$ & $\begin{array}{l}\text { „Am Anfang wird klar aufgeschrieben, was der Kunde will. } \\
\text { Was davon kann er schon, was sind die Ressourcen und wie } \\
\text { kann er sein Ziel erreichen?“ } \\
\text { „[Die Ziele] können Sie ja dann mit Schwerpunkten versehen } \\
\text { [...] und sagen: Du kannst nicht an } 25 \text { Punkten arbeiten, da } \\
\text { wirst du nie fertig, was ist denn jetzt das Wichtigste?“" }\end{array}$ \\
\hline $\mathrm{T}=$ Terminiert & Zielspezifität & $\begin{array}{l}\text { Für die Erreichung des spezifi- } \\
\text { schen Ziels wird eine Frist oder } \\
\text { ein Termin vereinbart }\end{array}$ & $\begin{array}{l}\text { „Der Coachee hat angefangen einen Projektkalender zu füh- } \\
\text { ren, damit er einen Überblick hat und sich selbst eine zeitlich } \\
\text { begrenzte Frist setzen kann.“ } \\
\text { „Ich sage: [...] schauen wir mal, welchen Zeithorizont wir } \\
\text { überhaupt haben, [...] was sind Meilensteine, an denen er auch } \\
\text { seinen Erfolg messen könnte?“ }\end{array}$ \\
\hline
\end{tabular}

1. Der Interviewpartner gibt eine Kurzbeschreibung des Coaching-Prozesses (Anliegen, Ablauf usw.).

2. Er trägt die wichtigsten Ereignisse im Zeitstrahl ein und schildert sie.

3. Rückspiegeln, Paraphrasieren und Zusammenfassen durch den Interviewer regt den Interviewpartner zur Präzisierung und Anreicherung seiner Schilderungen an.

4. Er zeichnet die Höhen und Tiefen des Prozesses im Zeitstrahl ein (auf diese Weise wird die Grundlage für die Tiefenexploration kritischer Ereignisse und deren Bewertungen durch den Interviewpartner geschaffen).

5. Explorierung der Höhen, Tiefen, Wendepunkte und weiterer wichtiger Aspekte des Coaching-Prozesses durch den Interviewer.

6. Rückspiegeln, Paraphrasieren und Zusammenfassen durch den Interviewer regt den Interviewpartner zur Präzisierung, Anreicherung und Bewertung geschilderter Ereignisse an.

7. Spezifisches Nachfragen durch den Interviewer.
Dieses Vorgehen bietet gegenüber anderen Interviewmethoden den Vorteil, dass es stark erzählfördernd und erinnerungsgenerierend ist, so dass Ereignislücken und unerwünschte Verzerrungen reduziert werden können.

Gegenüber Videobeobachtungen ermöglichen ZeitstrahlInterviews indes erwünschte Verzerrungen in der Form, dass die Interviewpartner bedeutende Ereignisse fokussieren, interpretieren und bewerten, irrelevante Ereignisse hingegen ausblenden. Des Weiteren konnten wir damit auch vor- und nachgelagerten Coaching-Phasen sowie sonstige unsichtbare und unbeobachtbare Vorgänge erheben, welche für den Verlauf und den Erfolg von Coachings kritisch sind. Hierzu gehören beispielsweise Reflexionen bei Coachs oder Klienten, Supervisionen und Weiterbildungsaktivitäten von Coachs oder die Gestaltung von Coaching-Rahmenbedingungen in Organisationen.

\subsection{Datenauswertung}

Die transkribierten Interviews wurden inhaltsanalytisch (Mayring 2010) und nach der Grounded Theory (Glaser 
Abb. 3 Wie SMART werden die Coaching-Ziele geklärt? (\% der Befragten; Poetschki und Wastian 2012)

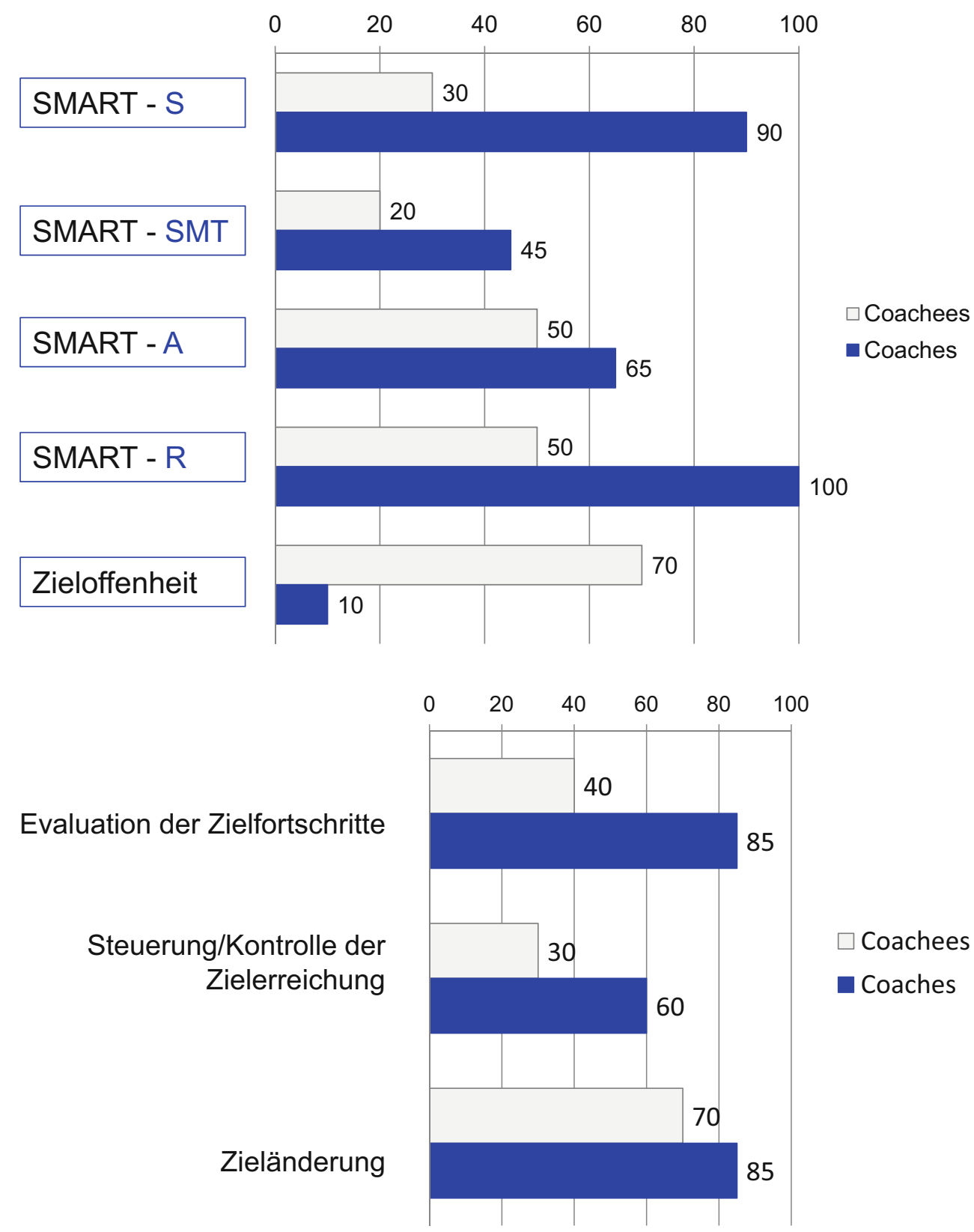

Abb. 4 Inwieweit erfolgte eine Evaluation, Steuerung/Kontrolle bzw. Änderung der Ziele? (\% der Befragten; Poetschki und Wastian 2012) und Strauss 2005; Strauss und Corbin 1996) mit Hilfe des Computerprogramms Atlas.ti ausgewertet. Dabei wurden die geschilderten Coach-Aktivitäten zum Arbeiten mit Zielen offen codiert. Daraus ließen sich folgende Code-Kategorien ableiten: a) die Zielklärung (Vorgehensweisen zur Exploration, Reflexion, Definition und Operationalisierung von Zielen), b) das Thematisieren von Zieländerungen, c) die Evaluation von Zielfortschritten (das Beobachten, Messen oder Hinterfragen von Veränderungen in Richtung des angestrebten Zielzustandes) und d) Maßnahmen, die laut Interviewpartner explizit der Steuerung und Kontrolle der Zielerreichung dienten (die Unterstützung und Kontrolle des Coachee bei der Umsetzung, z. B. Aktionspläne aufstellen, Ziele in Einzelschritte herunterbrechen, Durch- führung hinterfragen). Die Aussagen zur Zielklärung und Evaluation wurden dann theoriegeleitet entsprechend den Kriterien SMARTer Ziele codiert (siehe Definitionen und Beispielzitate in Tab. 1).

Weiterhin wurde analysiert, in welchen Phasen des Coaching-Prozesses und in welchen Sequenzen die Zielklärung erfolgte, um die Muster des Zielklärungsprozesses zu identifizieren. Hierzu wurden die Schilderungen der CoachAktivitäten sequenziell verknüpft und zusätzlich mit dem Zeitpunkt im Coaching-Prozess codiert, zu dem sie erfolgten (z. B. dritte Sitzung). Da die Coachings unterschiedlich lang dauerten - nämlich zwischen 2 bis 11 Sitzungen -, wurden die Zeitpunkte nach folgenden Phasen kategorisiert: Vor dem Coaching (Aussagen, welche den Zeitraum vor der 


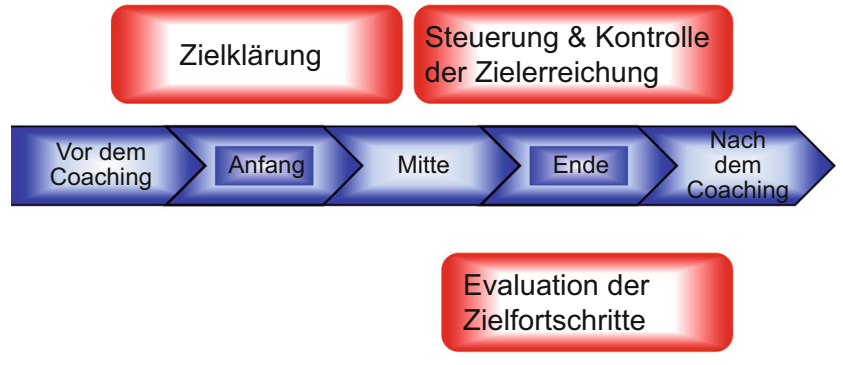

Abb. 5 Wann werden im Coaching-Prozess Ziele thematisiert? (Poetschki und Wastian 2012)

ersten Coaching-Sitzung betreffen, z. B. das Vorgespräch), Anfang - Mitte - Ende (Aussagen, welche die erste, die weiteren bzw. die letzte Coaching-Sitzung betreffen) und „Nach dem Coaching“ (Aussagen, welche Aktivitäten nach der letzten Sitzung betreffen, z. B. Follow-up-Gespräche).

Die Codes und Kategorien wurden schrittweise entwickelt, angepasst und validiert. Sie wurden im Forscherteam an so vielen Interviews getestet, diskutiert und angepasst, bis sie als valide befunden wurden. Neben der kommunikativen Validierung wurde zu den o.g. Kategorien anhand von fünf Interviews zudem die Interrater-Reliabilität erhoben. Ziel war eine Reliabilität $>0,75$ bei der Zuordnung der Codes bzw. Kategorien durch drei Mitglieder des Forscherteams. Die erhobene IR lag nach dem letzten Durchgang zwischen 0,81-0,92.

Anlässlich eines Coaching-Symposium mit CoachingExperten (Coachs, Personalentwickler, Coaching-Forscher) wurden außerdem die im folgenden Abschnitt dargestellten Ergebnisse im Rahmen von Workshops validiert und dienten als Grundlage für die Entwicklung eines CoachTrainings (,Eine Reise durch die Zielelandschaft“, Poetschki und Albert 2012).

\section{Ergebnisse}

Im Folgenden werden die Ergebnisse zu den Forschungsfragen vorgestellt.

\subsection{Wie wird der Weg zum Ziel geklärt, gesteuert und kontrolliert?}

Die Abb. 3 zeigt, inwieweit die befragten Coachs und Coachees Vorgehensweisen thematisierten, welche auf die Anwendung der SMART-Kriterien bei der Zielklärung schließen lassen. Dabei zeigt sich eine deutliche Diskrepanz zwischen den Schilderungen von Coachs und Coachees: $90 \%$ der Coachs beschrieben eine spezifische (= S) Zielklärung, während nur $10 \%$ zieloffen vorgingen, $\mathrm{d}$.h. die Ziele vage oder in einem Fall explizit gar nicht klärten. Dagegen nahmen nur $30 \%$ der Coachees eine spezifische Zielklärung

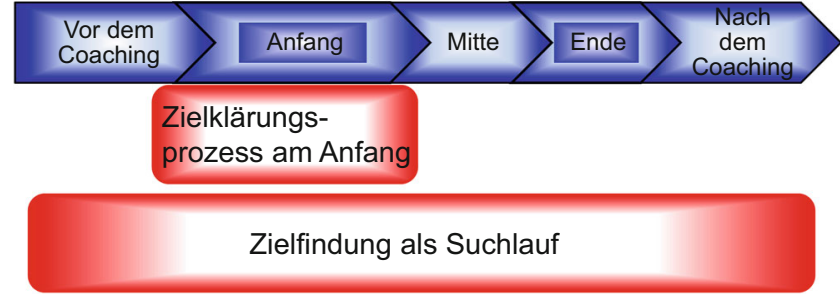

Abb. 6 Typische Muster der Zielklärung im Coaching-Prozess (Poetschki und Wastian 2012)

wahr, $70 \%$ beschrieben eine vage Zielklärung. Die Operationalisierung spezifischer, messbarer und terminierter (= SMT) Ziele schilderten nur $45 \%$ der Coachs (vs. $20 \%$ der Coachees). Alle Coachs, jedoch nur die Hälfte der Coachees beschrieben, dass die Realisierbarkeit $(=\mathrm{R})$ der Ziele hinterfragt wurden. Die Exploration der Zielakzeptanz (= A) wurde von $65 \%$ der Coachs (vs. 50\% der Coachees) thematisiert.

Die Abb. 4 stellt dar, dass Vorgehensweisen zur Evaluation der Zielfortschritte sowie zur Steuerung und Kontrolle der Zielerreichung von Coachs ebenfalls wesentlich öfter geschildert werden als von Coachees. Ähnlich häufig gaben Coachs und Coachees an, dass sich die Coaching-Ziele im Verlauf des Coachings änderten.

\subsection{Wann werden im Coaching-Prozess Ziele thematisiert?}

Abb. 5 zeigt, dass das Arbeiten mit Zielen den gesamten Coaching-Prozess beherrscht, wobei die Zielklärung nicht nur im Vorgespräch und in der ersten Sitzung (Phasen „Vor dem Coaching" und „Anfang") erfolgen muss (vgl. auch Abb. 6). Die Evaluation der Zielfortschritte sowie die Steuerung und Kontrolle der Zielerreichung reichen bis zum Coaching-Abschluss und manchmal darüber hinaus (in Form von Follow-up-Maßnahmen wie Treffen, Telefonate, Befragungen).

\subsection{Lassen sich typische Muster des Zielklärungs- bzw. Zielerreichungsprozesses erkennen?}

In Bezug auf zielbezogene Vorgehensweisen von Coachs ließen sich in den von uns untersuchten Coaching-Prozessen zwei typische Muster im Umgang mit Zielen identifizieren (siehe Abb. 6), die sich im Wesentlichen in der Zielklärung zeigen:

- Die Zielklärung zu Beginn des Coachings: Der Coach versucht explizit bereits im Vorgespräch oder in der ersten Sitzung eine SMARTe Zielklärung. Anhand der SMARTen Ziele evaluiert er die Zielfortschritte und 
steuert ggf. die Zielerreichung. Zu Beginn exploriert er dazu beispielsweise die Zielerreichung, im Verlauf oder auch am Schluss des Coachings anhand von Skalen und gibt dem Coachee Feedback zu Zielfortschritten, indem er ihm Veränderungen in den Skalenwerten aufzeigt.

- Die Zielfindung als „Suchlauf“ über den gesamten Coaching-Prozess hinweg: In diesem Fall stellt die Zielklärung eine wesentliche Coaching-Intervention dar, die sich als Aushandlungsprozess über das gesamte Coaching erstreckt. Das Coaching beginnt zieloffen. Die Ziele werden im Verlauf des Coachings jedoch permanent hinterfragt, bei Bedarf angepasst und zunehmend konkretisiert. Dabei sind Zielklärung und Intervention stark verwoben.

Lediglich einer der interviewten Coachs wich von diesen beiden typischen Mustern ab. Er ging völlig zieloffen vor, d. h. er nahm explizit zu keinem Zeitpunkt eine Zielklärung, was er mit seinem psychoanalytischen Ansatz begründete. Entsprechend erhob er auch keine Zielfortschritte und keine Zielerreichung, da er das Coaching als offenen Prozess verstand, bei dem zu Beginn selbst auf Vereinbarungen zum zeitlichen Umfang oder zur Anzahl der Sitzungen verzichtet wurde.

\section{Diskussion}

\subsection{Zentrale Fragestellungen und Ergebnisse}

Basierend auf qualitativen Forschungsmethoden wurde untersucht, a) wie im Coaching Ziele geklärt und Zielfortschritte gesteuert werden, b) wann Ziele zwischen den Beteiligten thematisiert werden und c) ob es typische Muster des Zielklärungs- bzw. Zielerreichungsprozesses gibt. Wie sich herausstellte, werden Coaching-Ziele zwar während des gesamten Coaching-Prozesses thematisiert, wobei sich bei der Zielklärung typische Muster zeigen (vorwiegend die Zielklärung am Anfang vs. die Zielfindung als Suchlauf über den gesamten Coaching-Prozess hinweg). SMARTe Ziele stellen zwar nach den Aussagen unserer Interviewpartner auch im Coaching einen wichtigen Erfolgsfaktor dar, doch werden sie überwiegend nicht spezifisch, messbar und terminiert geklärt. Zudem klaffen die Sichtweisen von Coachs und Coachees diesbezüglich deutlich auseinander.

Eine Begründung dafür, dass Coachs wesentlich häufiger als Coachees zielbezogene Maßnahmen und SMARTAspekte erwähnen, kann sein, dass die Coachs der befragten Coachees anders vorgingen als die von uns befragten Coachs. Es ist aber auch möglich, dass Coachs und Coachees eine andere Wahrnehmung für die SMARTe Zielklärung, -kontrolle und -erreichung haben. Als Laien können Coachees die systematischen Gesprächstechni- ken der Coachs vermutlich nur schwer erkennen. Dagegen spricht jedoch, dass die Klärung spezifischer, messbarer Ziele, mehr Transparenz und Feedback zu ihren Zielfortschritten der wichtigste Verbesserungswunsch einiger Coachees war (Graf und Wastian 2014, S. 22).

\subsection{Limitationen}

Zwar ist das Sample der vorliegenden Untersuchung vergleichsweise groß für eine qualitative Studie und bildet die Welt der Business Coachings valide ab, doch kann kein Anspruch auf Repräsentativität erhoben werden. Die Rekrutierung der Interviewpartner folgte Opportunitätsüberlegungen (Zugänglichkeit des Feldes, Reduzierung von Reisekosten). Zudem war es, bis auf einzelne Ausnahmen, aufgrund des schwierigen Feldzugangs nicht möglich, CoachCoachee-Paare für die Interviews zu gewinnen, was im Hinblick auf die Vergleichbarkeit der geschilderten CoachingProzesse und der Ergebnisse wünschenswert gewesen wäre.

Da die Datenerhebung mit Hilfe von Interviews erfolgte, unterliegen die Ergebnisse verschiedenen Verzerrungstendenzen. Seitens der Interviewer wurde die Darstellung der Coaching-Prozesse nicht durch einen stringenten Leitfaden vorgegeben, sondern orientierte sich mit klientenzentrierten Gesprächstechniken an den Schilderungen der Interviewpartner. Damit können zwar erwünschte Verzerrungen - nämlich die Fokussierung der Interviewpartner auf die aus ihrer Sicht kritischen Ereignisse und deren Bewertung - herbeigeführt werden, doch seitens des Interviewers besteht die Gefahr unerwünschter Verzerrungen. Zum einen sind Zeitstrahl-Interviews (Wastian und Schneider 2005) gesprächsmethodisch sehr anspruchsvoll, zum anderen muss der Interviewer die Welt seiner Gesprächspartner und die von ihnen geschilderten Zusammenhänge verstehen, um den Erzählfluss so zu lenken, dass die forschungsrelevanten Aspekte angemessen exploriert werden. In der vorliegenden Untersuchung wurden diese Risiken dadurch minimiert, dass die Interviewer neben einer intensiven Schulung in der Interviewmethode und einschlägigen Erfahrungen (Kenntnis klientenzentrierter Gesprächsführung, Praktika oder Berufstätigkeit in der Wirtschaft, in Coaching und/oder Beratung) auch Feedback zur Interviewführung erhielten (Reflexion der Aufzeichnungen im Team) und die ersten Interviews gemeinsam mit erfahrenen Kollegen durchführen konnten.

\subsection{Implikationen für die Forschung}

Um zu klären, ob die Anwendung der SMART-Kriterien und anderer zielbezogenen Vorgehensweisen von Coachs tatsächlich unzureichend erfolgt oder von den Coachees nicht wahrgenommen wird, bedarf es also weiterer Untersuchungen, bei denen Coaching-Prozesse sowohl beobach- 
tet, als auch interview-gestützt - wie hier mit der ZeitstrahlMethode (Wastian und Schneider 2005) - rekonstruiert werden. Zudem sollten zum jeweiligen Coaching-Prozess sowohl die Coach- als auch die Coachee-Perspektive erhoben werden, um eine Vergleichbarkeit der Sichtweisen zu gewährleisten.

Desweiteren sollten die Determinanten bzw. Effekte der beiden wesentlichen Zielklärungsstrategien - Zielklärung zu Beginn des Coachings oder als „Suchlauf“ über den Coaching-Prozess hinweg - untersucht werden. Es ist davon auszugehen, dass Coaches ihre Strategie entweder von ihren methodischen Präferenzen und Kompetenzen abhängig machen oder dass sie sich dabei an den Coachees und deren Anliegen orientieren. Im zweiten Fall stellt sich auch die Frage, bei welcher Art von Anliegen bzw. bei welchen Coachee-Merkmalen welche Strategie effektiver ist.

\subsection{Implikationen für die Praxis}

\subsubsection{SMART ist nicht immer zielführend}

Sowohl die Forschung zur Zielsetzung (vgl. Abschn. 2) als auch die Ergebnisse unserer Untersuchung verdeutlichen die Bedeutung der Zielklärung, der Steuerung und Kontrolle sowie der Evaluation von Zielfortschritten für einen erfolgreichen Coaching-Prozess. Jedoch sind sowohl bei der SMARTen Zielklärung als auch bei der Zielfindung als Suchlauf gewisse Hürden zu bedenken.

Die SMARTe Zielklärung hat den Vorteil - sofern der Coach sie beherrscht -, dass sie sehr stringent ein Ziel formuliert und dadurch im Verlauf des Coachings gut zu überprüfen ist. Dadurch kann aber das Problem entstehen, dass sich Coachs bzw. Coachees zu sehr auf dieses Ziel konzentrieren und möglicherweise übersehen, wenn während des Prozesses eine Zieländerung notwendig wird.

Es ist wichtig, dass der Coach die Akzeptanz (= A in der SMART-Formel) des Ziels regelmäßig überprüft, um Zieländerungen rechtzeitig zu erkennen. Sonst kann es sein, dass der Coachee sich nicht mit dem gesetzten Ziel identifiziert und nur mit mäßigem Einsatz daran arbeitet.

Eine weitere Hürde kann das Anliegen an sich sein. Eine SMARTe Klärung von Verhaltenszielen empfiehlt sich beispielsweise, wenn es um Fragen der Kompetenzentwicklung geht (Graf und Wastian 2014). In einigen Fällen erscheint es dagegen ratsamer den Coaching-Prozess erst einmal ohne die Definierung von Zielen anlaufen zu lassen. An dieser Stelle würde sich dann das Ziel innerhalb des Prozesses als sogenannter „Suchlauf“ ergeben, z. B. wenn der Coachee mit mehreren „Baustellen“ zum Coach kommt und zunächst herausgefunden werden muss, welches Anliegen überhaupt vorliegt.

Zusammenfassend lässt sich sagen, dass es bei der Frage, ob und wie ein SMARTer Zielprozess im Coaching statt- finden sollte, auf den Coachee, das Wissen und die Umstände über den methodischen Einsatz seitens des Coachs ankommt. Hier sollte individuell entschieden werden, ob der SMARTe Weg der Richtige ist.

\subsubsection{Handlungsempfehlungen für Coaching-Beteiligte}

Durch das Projekt „Qualitätssicherung im Coaching“ konnten über die o.g. Ausführungen hinaus Handlungsempfehlungen identifiziert werden, die sich u. a. auf die Zielklärung, auf die Steuerung und Kontrolle der Zielerreichung und auf die Evaluation von Zielfortschritten beziehen (vgl. die Untersuchungsergebnisse von Klimt und Wastian 2014; Schumacher und Wastian 2014). Sie zeigen, wie CoachingBeteiligte selbst zur Qualität von Coachings beitragen können und wie Coaching seitens der Organisation und der Personalentwicklung kommuniziert, vorbereitet und begleitet werden kann.

Forschungsergebnisse zur Zielsetzungstheorie (vgl. Abschn. 2) sprechen für SMART - aber: In der CoachingRealität erfolgt oft keine SMARTe Zielklärung, obwohl die Prozesse als erfolgreich gelten können. Dies entspricht auch den Befunden zur Effektivität (Theeboom et al. 2013) und zur hohen Akzeptanz von Coachings (Überblick: Greif 2008). Praktizierende Coachs sollten jedoch dahingehend sensibilisiert werden, dass Ziele in einem Coaching-Prozess ein wichtiger Faktor für einen erfolgreichen Ausgang sind. Das Vorgehen bei der Zielklärung sollte für Coachees transparent sein und die Bedeutung SMARTer Ziele für die Zielerreichung noch stärker herausgestrichen werden, da insbesondere spezifische und messbare Ziele von Coachees als wichtiger Verbesserungswunsch genannt wurde.

Sowohl in Zusammenhang mit der Klärung von Zielen als auch mit der Steuerung, Kontrolle und Evaluation der Zielfortschritte hat sich das Visualisieren und Dokumentieren als elementarer Wirkfaktor erwiesen (Klimt und Wastian 2014; Wastian 2015). Hierfür eignen sich beispielsweise Coaching-Tagebücher, Zielerreichungs-Skalen und das Festhalten wichtiger Fortschritte und Maßnahmen auf Flipchart. Sie ermöglichen Coach und Coachee das kontinuierliche Überprüfen und Feedback über den Stand der Zielerreichung. Für die Steuerung und Kontrolle ist auch das Zusammenfassen und Strukturieren des Coaching-Prozesses wichtig. Dabei sollten etappenweise oder nach jeder Sitzung Erkenntnisse bzw. Vorgehensweisen auf dem Weg zur Zielerreichung mit dem Coachee erörtert werden.

Im Falle der Veranlassung eines Coachings durch den Personalentwickler oder der Führungskraft des Coachees, sollten diese Personen nach Meinung der Coaching-Beteiligten in die Zielklärung - im Rahmen eines gemeinsamen Vorgesprächs - und in die Evaluation von Zielfortschritten einbezogen werden. Zum Abschluss, aber auch während des Coaching-Prozesses könnte der Coach dann 
mit dem Coachee und dessen Führungskraft Feedbackgespräche zu Zielfortschritten des Coachees führen. Dadurch sei es der Führungskraft möglich, den Entwicklungsprozess seines Mitarbeiters mit zu verfolgen und zu unterstützen (vgl. Schumacher und Wastian 2014).

Die Coachees selbst sollten sich nach Ansicht unserer Interviewpartner vor Beginn eines Coachings über ihre Coaching-Ziele Klarheit verschaffen. Sie sollten ein möglichst konkretes Anliegen haben und dem Coach mitteilen, was geändert und was beibehalten werden kann. Erfolgreiche Coachees hatten beispielsweise klare Vorstellungen darüber, zu welchem Handeln sie im Stande sind und was sie erreichen wollen. Anfänglich nur vage Ziele können jedoch auch erst im Coaching gemeinsam erarbeitet werden. Weiterhin halten es die von uns befragten Coachs ratsam, dass der Coachee relativierte, realistische Erwartungen an das Coaching und dessen Ergebnisse hat. Ihrer Meinung nach sollte der Coachee eine aktive Steuerungs- und Mitgestaltungsfunktion im Prozess übernehmen, beispielsweise durch die eigene Selbstreflektion oder das Hinterfragen hinsichtlich der Zielerreichung. Diese Empfehlungen können unserem Forschungsprojekt zufolge den Erfolg des Coaching-Prozesses unterstützen (vgl. Schumacher und Wastian 2014).

\section{Fazit}

Um der Forderung nach Qualität im Coaching nachzukommen, ist eine qualitativ hochwertige Aus- und Weiterbildung im Coaching notwendig. Da sich die von Greif (2008) angenommenen Wirkfaktoren bereits empirisch bestätigen ließen (vgl. u. a. Greif et al. 2012; Klimt und Wastian 2014; Poetschki und Wastian 2014; Triebel 2009), liegt es nahe, sie in der Aus- und Weiterbildung von Coachs zu vermitteln. Ziele sollten während eines Coaching-Prozesses geklärt und kontinuierlich überprüft werden, um sie bei Bedarf anpassen zu können und um die Zielerreichung zu unterstützen. Hier bietet sich die Zielsetzungstheorie von Locke und Latham (1990), z. B. die Anwendung der SMART-Kriterien, an. Zudem ist ein gezieltes Training denkbar, indem die hier aufgeführten Phasen eines Ziel-Prozesses im Coaching bearbeitet und geübt werden können.

Eine Grundlage stellten förderliche Verhaltensweisen bzw. Merkmale des Coachs (Klimt und Wastian 2014)(siehe auch die o. g. Handlungsempfehlungen) dar, um Instrumente zur Coach-Auswahl sowie zur Aus- und Weiterbildung von Coachs zu entwickeln. Sie können in der Weiterbildung vermittelt und als Kriterien des Lernerfolges in Übungsszenarien geprüft werden. Hierfür empfehlen sich differenzierte Kompetenzprofile für Coachs.
Interessenkonflikt M. Wastian und J. Poetschki geben an, dass kein Interessenkonflikt besteht.

Open Access Dieser Artikel wird unter der Creative Commons Namensnennung 4.0 International Lizenz (http://creativecommons.org/ licenses/by/4.0/deed.de) veröffentlicht, welche die uneingeschränkte Nutzung, Verbreitung und Wiedergabe für beliebige Zwecke erlaubt, sofern Sie den/die ursprünglichen Autor(en) und die Quelle ordnungsgemäß nennen, einen Link zur Creative Commons Lizenz beifügen und angeben, ob Änderungen vorgenommen wurden.

\section{Literatur}

Flanagan, J.C. (1954). The critical incident technique. Psychological Bulletin, 51, 327-358.

Glaser, B. G., \& Strauss, A.L. (2005). Grounded Theory. Strategien qualitativer Forschung (2. Aufl.). Bern: Verlag Hans Huber.

Graf, E.-M., \& Wastian, M. (2014). Coaching macht (Hoch)Schule, wenn ...: Erkenntnisse und Empfehlungen zur Qualitätssicherung im Coaching. Wirtschaftspsychologie, 2, 16-26.

Grant, A. M., Passmore, J., Cavanagh, M. J., \& Parker, H. (2010). The state of play in coaching today: a comprehensive review of the field. International Review of Industrial and Organizational Psychology, 25, 125-167.

Grawe, K., Donati, R., \& Bernauer, F. (1994). Psychotherapie im Wandel. Von der Konfession zur Profession. Bd. 4. Göttingen: Hogrefe.

Greif, S. (2008). Coaching und ergebnisorientierte Selbstreflexion. Theorie, Forschung und Praxis des Einzel- und Gruppencoachings. Göttingen: Hogrefe.

Greif, S., Schmidt, F., \& Thamm, A. (2012). Warum und wodurch Coaching wirkt. Ein Überblick zum Stand der Theorieentwicklung und Forschung über Wirkfaktoren. Organisationsberatung Supervision - Coaching, 19(4), 375-390.

Johnson, S. K., Garrison, L.L., Hernez-Broome, G., Fleenor, J. W., \& Steed, J. L. (2012). Go for the goal(s): Relationship between goal setting and transfer of training following leadership development. Academy of Management Learning \& Education, 11(4), 555-569.

Jones, R. J., Woods, S. A., \& Guillaume, Y.R.F. (2015). The effectiveness of workplace coaching: A meta-analysis of learning and performance outcomes from coaching. Journal of Occupational and Organizational Psychology.

Klein, H.J., Wesson, M.J., Hollenbeck, J.R., \& Alge, B.J. (1999). Goal commitment and the goal-setting process: Conceptual clarification and empirical synthesis. Journal of Applied Psychology, 84(6), 885-896.

Klimt, S., \& Wastian, M. (2014). Ein Kompetenzkatalog für Coaches: Grundlage zur Qualitätssicherung bei der Auswahl sowie der Aus- und Weiterbildung von Coaches. „Coaching heute - Zwischen Königsweg und Irrweg“, Erding, February, 20-21. Paper presented at the Coaching-Kongress an der HAM

Künzli, H. (2009). Wirksamkeitsforschung im Führungskräfte-Coaching. Organisationsberatung - Supervision - Coaching, 16, $1-15$.

Locke, E. A., \& Latham, G.P. (1990). A theory of goal setting \& task performance. Englewood Cliffs, NJ: Prentice Hall.

Locke, E.A., \& Latham, G.P. (2002). Building a practically useful theory of goal setting and task motivation. American Psychologist, 57(9), 705-717.

Mayring, P. (2010). Qualitative Inhaltsanalyse - Grundlagen und Techniken. Bd. 11. Weinheim: Beltz. aktualisierte und überarbeitete Auflage

Neubert, M. J. (1998). The value of feedback and goal setting over goal setting alone and potential moderators of this effect: A meta-analysis. Human Performance, 11(4), 321.

Passmore, J., \& Fillery-Travis, A. (2011). A critical review of executive coaching research: A decade of progress and what's to come. Coa- 
ching: An International Journal of Theory, Research and Practice, 4(2), 70-88.

Poetschki, \& Albert, J. (2012). Eine Reise durch die Zielelandschaft, München, 9. November. Paper presented at the Symposium für Qualitätssicherung im Coaching

Poetschki, J., \& Wastian, M. (2012). Coaching-Ziele klären und erreichen - was ist wirklich SMART? München, November 9. Paper presented at the Symposium für Qualitätssicherung im Coaching

Poetschki, J., \& Wastian, M. (2014). Coaching-Ziele klären und erreichen - was ist wirklich SMART? „Coaching heute - Zwischen Königsweg und Irrweg“, Erding, February, 20-21. Paper presented at the Coaching-Kongress an der HAM

Rogers, C.R. (2005). Die klientenzentrierte Gesprächspsychotherapie (17. Aufl.). Frankfurt: Fischer.

Schmidt, K.-H., \& Kleinbeck, U. (2006). Führen mit Zielvereinbarung. Göttingen: Hogrefe.

Schumacher, K., \& Wastian, M. (2014). Erfolgreiches Coaching - was können Coachees, deren Führungskräfte und Personalentwickler/ innen dazu beitragen? „Coaching heute - Zwischen Königsweg und Irrweg“", Erding, February, 20-21. Paper presented at the Coaching-Kongress an der HAM

Seijts, G. H., Latham, G. P., Tasa, K., \& Latham, B. W. (2004). Goal setting and goal orientation: An integration of two different yet related literatures. Academy of Management Journal, 47(2), 227-239.
Strauss, A., \& Corbin, J.M. (1996). Grounded Theory. Weinheim: Beltz.

Theeboom, T., Beersma, B., \& Van Vianen, A. E. M. (2013). Does coaching work? A meta-analysis on the effects of coaching on individual level outcomes in an organizational context. The Journal of Positive. Psychology, 9(1), 1-18.

Triebel, C. (2009). Kompetenzbilanzierung als psychologische Intervention. Wirkfaktoren und Wirkprinzipien in Laufbahnberatung und Coaching. Dissertation, Universität der Bundeswehr, München.

Wastian, M. (2015). Wirkfaktoren auch im E-Coaching nutzen: Was passiert in der „Black Box“ des virtuellen Coaching-Prozesses? In H. Geißler, \& R. Wegener (Hrsg.), Bewertung von Coachingprozessen (S. 299-321). Wiesbaden: Springer.

Wastian, M., Braumandl, I., \& Weisweiler, S. (2015). Führung und Mikropolitik in Projekten. Der psychologische Faktor im Projektmanagement. Wiesbaden: Springer.

Wastian, M., \& Schneider, M. (2005). Zeitliche Aspekte des Projektmanagements: Die Bedeutung von Phasen, Laufzeiten, Impulsen, Ups und downs. Wirtschaftspsychologie, 7(3), 28-35.

Winkler, B., \& Hofbauer, H. (2010). Das Mitarbeitergespräch als Führungsinstrument. Handbuch für Führungskräfte und Personalverantwortliche (4. Aufl.). München: Carl Hanser. 